\title{
PHOTOIONIZATION OF SOME ALKALI VAPORS
}

\author{
By F. L. Mohler and C. Boeckner
}

ABSTRACT

Relative measurements of photosensitivity as a function of wave length have been made by the space-charge method, and absolute values at favorable wave lengths have been measured by a direct method.

Caesium.-The shape of the sensitivity curve is similar to published results and independent of pressure. The photoionization is proportional to the pressure giving an atomic absorption coefficient $k$ of $2.3 \times 10^{-10}$ at the principal series limit.

Rubidium.-The sensitivity curve is similar to caesium and the value of $k$ is $1.1 \times 10^{-10}$ at the limit.

Potassium.-The sensitivity is low at the limit $2856 \mathrm{~A}$ and rises rapidly from 2600 to $2200 \mathrm{~A}$.

Absorption of caesium.-A rough measurement at low pressure gave a value of $3 \times 10^{-10}$ at $3100 \mathrm{~A}$, but with an uncertainty of the same magnitude as the effect measured.

Recombination of caesium.-An estimate of the value of $k$ for caesium, based on measurements of the intensity of recombination radiation, gives the same magnitude as the photoionization value.

\section{CONTENTS}

I. Introduction

II. Method and apparatus.

III. Results

1. Caesium

1. Caesium

2. Rubidium_._- 310

3. Potassium_...

4. Absorption measurements with caesium vapor

IV. Conclusions

\section{INTRODUCTION}

The absorption of alkali vapors for radiation of a frequency greater than the principal series limits and the consequent ionization of the atoms has been studied by a variety of methods with rather contradictory and puzzling results. Careful measurements of the absorption of sodium and potassium vapor have been made by Harrison ${ }^{1}$ and Ditchburn ${ }^{2}$ and Trumpy. ${ }^{3}$ Harrison found that the continuous

8 Trumpy, Zeits. f. Phys., 47, p. 804; 1928. 
absorption was a maximum near the sodium limit and dropped gradually toward the violet, but he concluded from other results that a large and unknown part of this absorption came from molecular aggregates, presumably $\mathrm{Na}_{2}$. Trumpy's absorption curves show a sharper maximum and are interpreted to be of atomic origin. Ditchburn attempted to separate molecular and atomic absorption in potassium from analysis of measurements at different pressures, using the line absorption as a measure of the atomic concentration, but he found that the form of the resulting curves depended greatly on the pressure. In some cases it dropped and then rose in going from the limit toward the violet while in other cases there was a continual rise. He estimated the atomic absorption coefficient at the limit as $10^{-19}$.

Measurements of the ionization produced by radiation offer the advantage that much lower pressures can be used. Lawrence ${ }^{4}$ and Williamson ${ }^{5}$ have studied the photoionization of potassium by illuminating a jet of vapor. The former found that radiation on the red side of $2600 \mathrm{~A}$ was ineffective, while beyond this point the efficiency increased rapidly. Williamson found a small effect beginning near $3100 \mathrm{~A}$ in addition to the increase beyond $2600 \mathrm{~A}$. The limit is at 2856 A. Figure 5 includes these results.

Measurements in caesium have given entirely different results. Mohler, Foote, and Chenault ${ }^{6}$ have used the effect of positive ions on a thermionic current limited by negative space charge to detect photoionization. This method and the vapor-jet method do not yield absolute values. Subsequently, Little ${ }^{7}$ has made direct measurements in vapor under equilibrium conditions. Both results show a sharp maximum accurately at the limit with a minimum near $2700 \mathrm{~A}$ and a gradual increase beyond this point. (See Fig. 2.) The last feature has been questioned in a recent note. ${ }^{8}$ Little gives the atomic absorption coefficient as $2.5 \times 10^{-21}$ at the limit which is surprisingly low.

In a recent paper ${ }^{9}$ the authors derived the relative probability of recombination of caesium ions and electrons as a function of the electron speed from the intensity distribution in the continuous emission band beyond the subordinate series limit. From this, by the principal of detailed balance, it was computed that the continuous absorption of atoms in the $2 P$ state varied as $\lambda^{4}$, a decrease much slower than that shown by the photoionization measurements for the $1 S$ state.

These results are in marked contrast with absorption measurements in the X-ray range where absorption always begins at the limit and

\footnotetext{
${ }^{4}$ Lawrence, Phil. Mag., 50, p. 345; 1925.

5 Williamson, Proc. Nat. Acad., 14, p. 793; 1928.

6 Mohler, Foote, and Chenault, Phys. Rev., 27, p. 37; 1926.

7 Little, Phys. Rev., 30, p. 109; 1927; and correction note, p. 963.

${ }^{8}$ Lawrence and Edlefson, Phys. Rev., 33, p. 265; 1929.

๑ Mohler and Boeckner, B. S. Jour. Research, 2 (R. P. 46), p. 489; 1929.
} 
decreases as $\lambda^{3}$. (The photoionization would vary as $\lambda^{4}$.) In view of these discrepancies, it seemed worth while to make absolute and relative measurements of photoionization for several of the alkali vapors using the same procedure for each. This paper includes measurements with caesium, rubidium, and potassium, though only relative measurements were made in the last case. The absolute measurements in caesium vapor were made with the cooperation of W. W. Coblentz and R. Stair and have been described in a joint note. $^{10}$ The material has been included in the present paper for completeness.

\section{METHOD AND APPARATUS}

From the definition of the atomic absorption coefficient $k(\nu)$ the amount of energy $E$ absorbed from a beam of energy flux $J(\nu)$ in a length $\imath$ is

$$
E=J(\nu) N p l \hbar(\nu)
$$

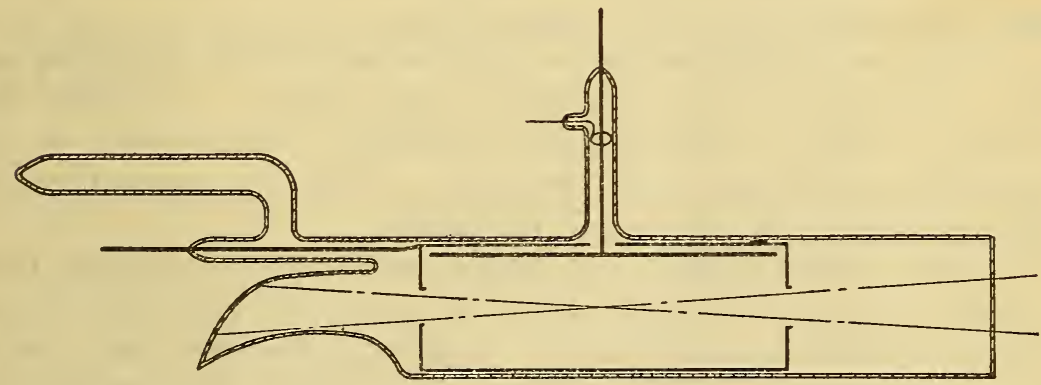

FigdRE 1.-Tube for direct measurement of photoionization

where $N$ is the number of atoms per $\mathrm{cm}^{3}$ at unit pressure and $p$ is the pressure. The number of ions produced per second $I / e$ (current/electron charge) will equal the number of quanta absorbed $E / h \nu$, so that

$$
k(\nu)=I h \nu / e N p l J(\nu)
$$

The sensitivity $I / J(\nu)$ is thus proportional to $k(\nu) / \hbar \nu$ or to Einstein's probability of absorption, $B$.

The determination of $k(\nu)$ is conveniently made in two parts-a measurement of the relative sensitivity at different wave lengths and an absolute measurement of $k$ at a favorable wave length. For the first purpose the space-charge method was selected because of its high sensitivity and the entire absence of surface effects. Absolute measurements were made under conditions such that the radiation flux could be definitely determined. It was not essential to use monochromatic radiation provided the spectral intensity distribution was known.

10 Mohler, Boeckner, Stair, and Coblentz;'Sci., 69, p. 479; 1929. 
Figure 1 illustrates the ionization chamber used for the direct measurement of the photoionization. One electrode is a cylinder $8 \mathrm{~cm}$ long and $3 \mathrm{~cm}$ in diameter with holes $1 \mathrm{~cm}$ in diameter at each end. The other is a plate nearly as long as the cylinder and $1 \mathrm{~cm}$ wide. Electrodes were platinum and the containing tube quartz. The beam of light focussed near the center of the cylinder was small enough, so that it passed through the holes without striking either. The cylinder was made positive and the plate negative to further reduce the possibility of photo-electric emission from electrode surfaces. Guard rings prevented any surface leak to the plate, but at an operating temperature of $220^{\circ} \mathrm{C}$. there is a thermionic emission of the order of $10^{-9} \mathrm{amp}$. Under steady temperature conditions this gave no trouble. Applied voltages of from 2 to 10 volts were required to secure a saturation ion current, and frequent check measurements were made to be certain that the operating voltage was adequate. Currents were measured by a Leeds \& Northrup galvanometer at a sensitivity of about $5 \times 10^{-11}$ amp. per mm.

For the work with caesium we used radiation from a mercury arc (110 volt Uviarc) passed through suitable filters. A circular diaphragm in front of the arc gave a focal spot small enough to fall entirely on a Coblentz disk thermopile, so that the energy flux was given directly by the thermopile calibration.

In other measurements, both relative and absolute, radiation from a mercury arc and from iron and brass arcs in air was resolved by a Bausch \& Lomb monochromator. A direct comparison was made of the photoionization and the reading of a Coblentz linear thermopile at the exit slit of the monochromator. This was corrected by later observing the thermopile reading at the position of the tube. Slit widths ranging from $20 \mathrm{~A}$ to $60 \mathrm{~A}$ at $3100 \mathrm{~A}$ were used. With the $60 \mathrm{~A}$ slit the 3130 lines of the mercury arc gave a radiation flux of about 100 ergs $\mathrm{sec}^{-1}$ while an 8 -amp. iron arc gave three to seven times this energy at 2750,2550 , and $2350 \mathrm{~A}$. The brass arc gave a comparable intensity near $2200 \mathrm{~A}$. Thermopiles and galvanometer were calibrated from time to time under operating conditions by means of a radiation standard. ${ }^{11}$

Measurements of the relative sensitivity at different wave lengths were made by. the space-charge method following the procedure described by Foote and Mohler. ${ }^{12}$ A hairpin cathode near the axis of a cylindrical anode is operated so that the current is limited by the space charge to a small fraction of the saturation current. The presence of positive ions increases the current; and, by balancing out the dark current, changes of $10^{-9} \mathrm{amp}$. can be measured. With caesium in the tube the radiation gave a current change of the order

11 Coblentz, B. S. Sci. Paper No. 227.

12 Foote and Mohler, Phys. Rev., 26, p. 195; 1925. 
of $10^{-5}$ amp., and when the effect is as large as this the current ceases to be proportional to the intensity. It was necessary to determine the current-intensity relation by measuring the effect produced when a series of wire gauze screens of known transmission were placed in the light beam.

Tubes were sealed off from the pumps after distilling in the alkali metal and thoroughly outgassing. In operation, the body of the tube was held at a fairly steady temperature of about $220^{\circ} \mathrm{C}$. while a small side tube containing the alkali metal was held at a lower temperature by a separate heater. This reduces the error in determining the vapor pressure; but even so, uncertainties in vapor-pressure data and in our temperature measurements make $p$ the most uncertain quantity in equation (1).

\section{RESULTS}

\section{CAESIUM}

Vapor pressures were computed from an equation, based on values by Kroner; ${ }^{13}$

$$
\log p=-\frac{3966}{T}+7.1650
$$

Relative measurements of the sensitivity were made with monochromatic radiations from mercury, iron, and brass arcs. The mercury lines on the red side of 3130 A give no effect while the 3130 group gives by far the greatest effect of all the lines. Because of the sensitivity of the space-charge method the precision was limited solely by the thermopile measurements and the inherent unsteadiness of air arcs Figure 2 gives these results and, for comparison, the published curves of Little ${ }^{14}$ and of Mohler, Foote, and Chenault. ${ }^{15}$ Repeated measurements in the range 2750 to $2200 \mathrm{~A}$ confirm the upward trend of the sensitivity curve at the short wave length end. Measurements with caesium at $180^{\circ} \mathrm{C}$. (vapor pressure $0.03 \mathrm{~mm}$ ) and at $115^{\circ} \mathrm{C}$. $(0.001 \mathrm{~mm})$ gave curves of identical shape.

The best absolute measurements were obtained with radiation from the mercury arc passed through a water cell and Corning purple glass G986 A. The transmitted light contains the lines 4047, 3650, 3300, 3130,3010 , and 2967 A. Addition of a piece of ordinary window glass removes 3130 and the lines of shorter wave length. It reduces the photoionization to zero and the thermopile readings to about 50 per cent. From energy measurements with the monochromator and the curve of Figure 2 we found that about 31 per cent of the radiation transmitted by the purple glass and 78 per cent of the photoionization

13 Rowe, Phil. Mag., 3, p. 534; 1927. (A critical summary of vapor tension data for all alkalies.)

14 See footnote 7, p. 304.

[15 See footnote 6, p. 304 
came from $3130 \mathrm{~A}$. Corrections for absorption in the tube and furnace windows were made. Figure 3 is a plot of the photoionization as a function of the vapor pressure over the temperature range $146^{\circ}$ to $201^{\circ} \mathrm{C}$. The effect is proportional to the pressure within experimental error. This fact and the absence of any effect for radiation on the red side of 3130 support the conclusion that surface photoelectric effect has been eliminated.

The caesium used in most of the work did not have a sharp melting point. The experiment was repeated with a carefully prepared sample which melted sharply near $28^{\circ} \mathrm{C}$. and one run gave the same value as



FIgURE 2.-Photo-electric sensitivity of caesium vapor

$I$, circles are based on measurements with a mercury arc, crosses on radiation from iron and brass arcs. II, is from published data of Mohler, Foote, and Chenault; III, from data of Little

before. Then, apparently a small air leak developed, and somewhat lower values were obtained though the oxidation of the caesium was barely visible. On the basis of the slope of the line in Figure 3 the value of $k$ at 3130 is $1.85 \times 10^{-19}$. Measurements with a filter of silvered quartz which transmitted only the 3130 group and with the monochromator checked this value but with somewhat greater experimental error. From the value of $k$ at 3130 and the sensitivity curve of Figure 2 we conclude that $k=2.3 \pm .2 \times 10^{-19}$ at the limit $3184 \mathrm{~A}$.

For the first time we are able to give a figure for the efficiency of the space-charge method. In the tube used in these experiments a 


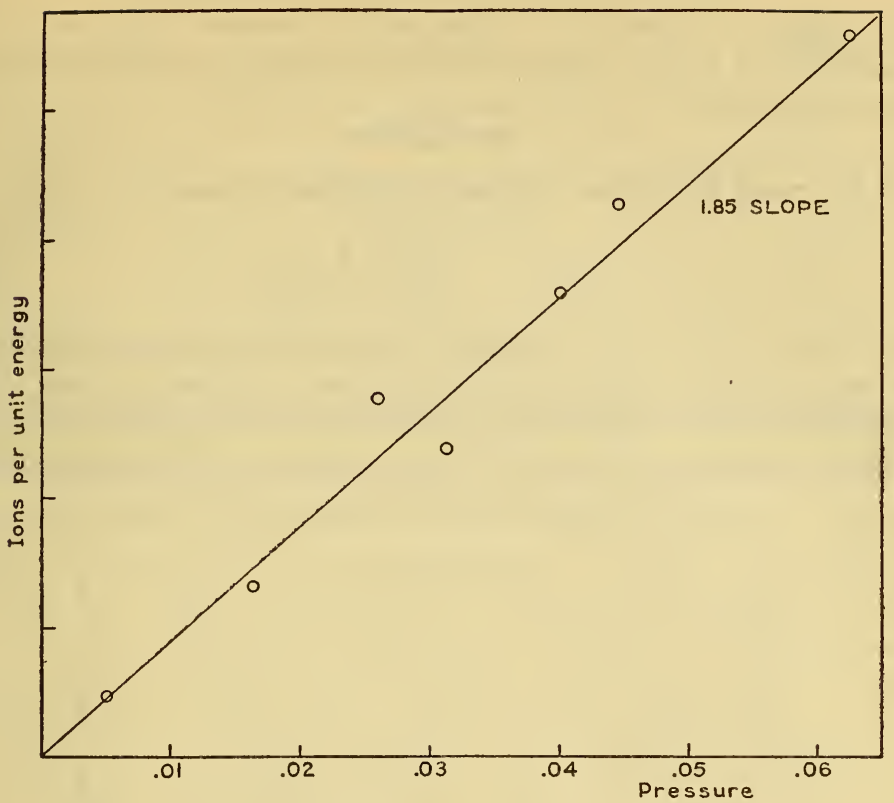

FiguRe 3.-Photoionization in caesium vapor as a function of the pressure

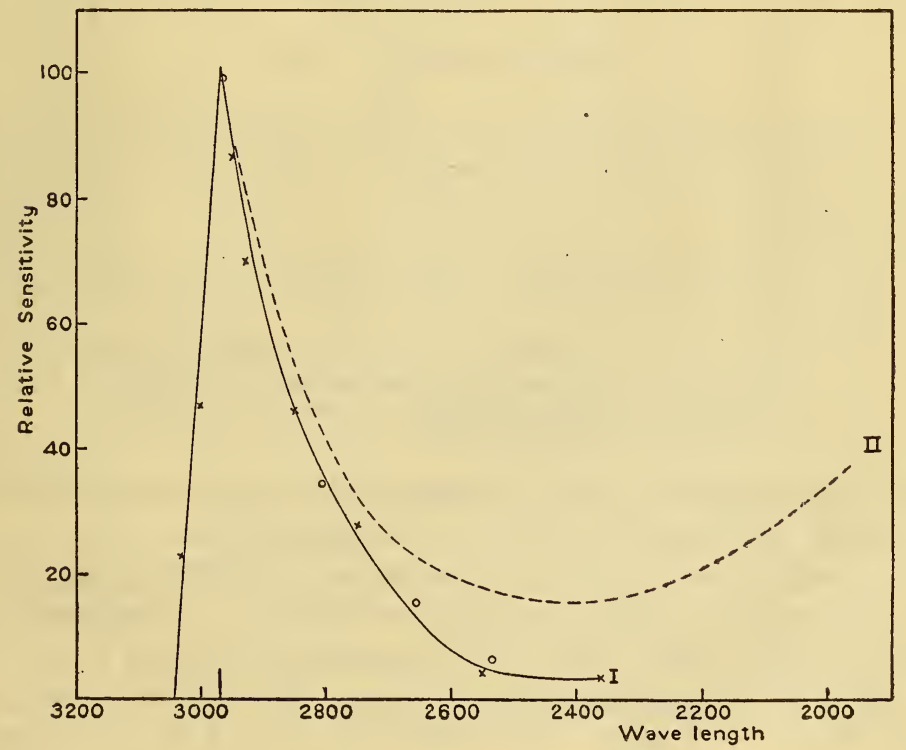

Figure 4.-I Photo-electric sensitivity of rubidium vapor

Circles are from measurements with a mercury arc, crosses from measurements with an iron arc. The limit is at $2968 \mathrm{~A}$. II is Curve $I$ for caesium with the wavelength scale shifted to bring the limits together 
current change of about $10^{4}$ times the ion current could be obtained with caesium at $180^{\circ} \mathrm{C}$. while at $110^{\circ} \mathrm{C}$. the amplification was about ten times as great.

\section{RUBIDIUM}

We have used the vapor tension equation of Scott, ${ }^{13}$

$$
\log p=-\frac{4209}{T}+7.331
$$

Figure 4 shows the relative sensitivity at different wave lengths based on the space charge effect of monochromatic radiation from the mercury and iron arcs. The mercury line 2967 falls within an Angstrom of the limit and gives by far the biggest effect of all the mercury lines.

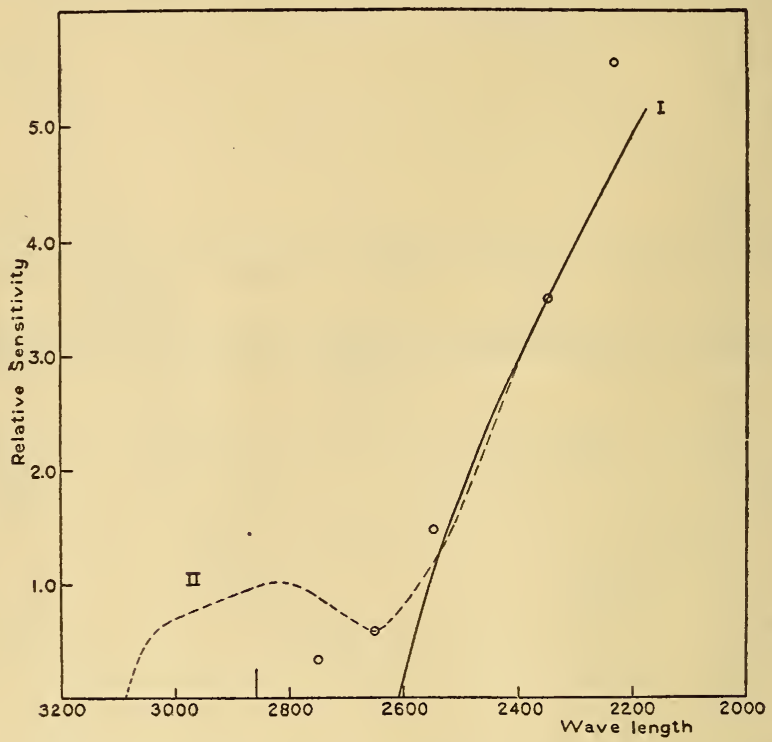

FigdRe 5.-Photo-electric sensitivity of potassium vapor

Circles give our measurements with iron and brass arc radiation. I and $I I$ are from published results by Lawrence and Williamson, respectively. The limit is at $2856 \mathrm{~A}$

A plot of the caesium sensitivity curve (II) reduced to the same wave length scale is included in Figure 4 for comparison.

The absolute value of the absorption coefficient was based on measurements at a single temperature $196^{\circ} \mathrm{C}$., corresponding to a pressure of $0.023 \mathrm{~mm}$, using monochromatic radiation from the iron arc. At $2750 \mathrm{~A}$ a radiation flux of about $360 \mathrm{ergs} \mathrm{sec}^{-1}$ gave an ion current of $4 \times 10^{-10} \mathrm{amp}$. The mean value of $k$ at 2750 was $2.9 \times$ $10^{-10}$ and from figure 4 we compute $k=1.1 \times 10^{-19}$ at the limit $2968 \mathrm{~A}$.

\section{POTASSIUM}

The efficiency of the space-charge method was low for potassium, but the radiation from iron and brass arcs gave an effect that was easily measured for wave lengths shorter than 2800 A. Figure 5 
shows our relative values in comparison with results of Williamson ${ }^{16}$ and of Lawrence ${ }^{17}$ obtained by the vapor-jet method. In our experiments the potassium was at $200^{\circ} \mathrm{C}$. corresponding to a vapor pressure of about $0.005 \mathrm{~mm}$. The potassium limit is at $2856 \mathrm{~A}$. The results are in striking contrast to the sensitivity curves of caesium and rubidium. The radiation at $2750 \mathrm{~A}$ is very intense, so that the low sensitivity near the limit could be measured with fair precision. Absolute measurements were not attempted.

\section{ABSORPTION MEASUREMENTS WITH CAESIUM VAPOR}

A direct measurement of the absorption coefficient of caesium rapor for the 3130 line of mercury was attempted, though the apparatus was not well adapted to the experiment. A quartz absorption cell $30 \mathrm{~cm}$ long was kept at about $230^{\circ} \mathrm{C}$., while an appendix containing caesium was successively heated and cooled. The space-charge tube was used to measure the intensity. The best series of readings with caesium heated to $218^{\circ} \mathrm{C}$. and cooled to $100^{\circ} \mathrm{C}$. gave a change of 2.4 per cent. This corresponds to a value of $k=3 \times 10^{-19}$ at $3130 \mathrm{~A}$. Other readings ranged from 1.8 to $6 \times 10^{-19}$, but accidental fluctuations were always of the same magnitude as the absorption effect. The photoionization method gave $k=1.85 \times 10^{-19}$ at $3130 \mathrm{~A}$.

\section{CONCLUSIOINS}

As concerns the shape of the photosensitivity curves there are only minor discrepancies between results obtained by different methods and observers. For caesium and rubidium the curves drop sharply from the limit to a value of 15 and 5 per cent, respectively, at $600 \mathrm{~A}$ beyond the limit; for potassium the sensitivity is low at the limit and rises toward the violet. It has been suggested that the former type of curve is characteristic of atomic vapor and the latter of molecular vapor. ${ }^{18}$ If so, the gradual rise at the violet end of the caesium curve and practically the entire effect in potassium may be of molecular origin. It is interesting that our measurements in stagnant potassium vapor at $0.006 \mathrm{~mm}$ give nearly the same results as were obtained by Lawrence ${ }^{19}$ and Williamson ${ }^{20}$ in jets issuing from a furnace at $400^{\circ} \mathrm{C}$. (partial pressure $4.5 \mathrm{~mm}$ ), for it has been suggested that this type of curve was characteristic of the jet method. These results are not inconsistent with the form of the absorption curves found by Ditchburn. ${ }^{21}$

${ }^{16}$ See footnote 5, p. 304.

17 See footnote 4, p. 304 .

18 See footnote 4, p. 304.

19 See footnote 4, p. 304.

20 See footnote 5, p. 304.

${ }^{21}$ See footnote 2, p. 303. 
The upward slope on the short wave length side of the caesium sensitivity curve may depend on impurities, for different observers are not in agreement on this, but the abnormal sensitivity at $2537 \mathrm{~A}$, found by Lawrence and Edlefson ${ }^{22}$ and ascribed to mercury vapor, was absent in this work. The fact that the form of our curves was quite independent of pressure is hard to understand if either caesium molecules or impurities influence the observations.

There is a surprising discrepancy between the absolute values of $k$ for caesium. Our experimental magnitudes are comparable with those of Little, ${ }^{23}$ except as concerns the observed ion current, and for that his values are of the order of one-fiftieth of ours for the same temperature. A low value can be obtained if insufficient caesium is in the tube or if there is a slight air leak. Little's measurements were in many respects more careful than ours, but various control experiments should rule out the possibility of a mistake in order of magnitude in our result.

There is a considerable variation in vapor-tension data of different observers sufficient to change the value of $k$ for caesium by a factor of two. Experimental values ${ }^{24}$ are given both for low pressures and high pressures with no measurements in the intermediate ranges we have used. Plots of log $p$ versus $1 / T$ give nearly parallel lines for different sets of observations, so that the best line through all sets has a slope quite different from all observed slopes. We found that values of $k$ computed from this mean line showed a systematic variation with pressure which was removed when we used values of pressure based on any single set of observational data. Because of the uncertainty in pressure the difference in the values of $k$ for caesium and rubidium has little significance.

The direct-absorption measurement in caesium is of interest in spite of its inaccuracy, for it indicates that the efficiency of photoionization is at least of the order of unity The existence of molecules with absorption limits on the short wave length side of the atomic limit would cause absorption without ionization superposed on the photo-electric absorption near the limit. In view of the possibility that there are such molecules it is important to know whether there are enough of them to seriously alter the atomic effect, for then the value of $k$ has little significance. We conclude that this is not the case in caesium vapor at $0.1 \mathrm{~mm}$.

The paper by the authors on recombination spectra of ions and electrons ${ }^{25}$ includes data for the estimation of the effective cross section $q$ of a caesium ion for recombination into the $2 P$ level. These

${ }_{22}$ See footnote 8, p. 304.

${ }^{23}$ See footnote 7, p. 304 .

${ }^{24}$ See footnote 13, p. 307.

${ }_{25}$ See footnote 9, p. 304. 
give a value $q=6.2 \times 10^{-21}$ for electrons with a velocity corresponding to 0.19 volt. (There is a numerical error in the published value.) By the principle of detailed balance

$$
\frac{k(\nu)}{q}=\frac{1}{a} \frac{m^{2} v^{2} c^{2}}{2 h^{2} \nu^{2}}
$$

where $m$ and $v$ are electron mass and speed and $\nu$ is the frequency at an interval beyond the limit corresponding to $v$. The a-priori probability, $a$, is taken as 3 for the superposed effect of the two $P$ levels. This gives $k=3 \times 10^{-17}$ near the $2 P$ limit for excited atoms in the $2 P$ state. Beyond the $1 S$ limit the intensity of the recombination light is of the order of 1 per cent of that beyond $2 P$. This makes $q=1.5 \times 10^{-23}$ for 0.19 volt electrons recombining into $1 S$ and $k=0.5 \times 10^{-19}$. The result involves values for the ion and electron concentrations which are quite uncertain, so that an agreement in order of magnitude with the observed value of $2.3 \times 10^{-19}$ is all that can be expected. We have noted in the introduction that the computed variation of $k$ with wave length for atoms in the $2 P$ state was very different from that observed for atoms in the normal $1 S$ state. It is desirable that the recombination measurements be extended and improved in absolute value so as to test more rigorously the applicability of the principle of detailed balance to this class of phenomena.

It is of interest to compare the absorption in the continuous region with data on line absorption. The significant datum in the case of an absorption line is the integral of the absorption across the width of the line, and in practice it is usually necessary to broaden the line by pressure or some other means to make the width measurable and to reduce the absorption at the center. Sugiura ${ }^{26}$ has shown that the integrated absorption over a frequency interval including one or more lines will approach in value the integral over an equal interval beyond the limit when the intervals are taken close to the limit. Waible ${ }^{27}$ has recently published measurements for the higher series lines in caesium with lines broadened by high vapor pressure (10 to 30 $\mathrm{mm}$.) and gives values of $\int k d \nu$ for lines from the fifth to the fourteenth, inclusive. If intervals $\Delta v$ are chosen equal to the successive intervals between lines so as to include one line in each interval, one finds that

$$
\frac{\int k d v}{\Delta \nu}=0.4 .8 \pm 0.01 \times 10^{-19}
$$

for all lines from the eighth to the fourteenth. This constancy is a consequence of the approximations that both the integral and $\Delta \nu$ are inversely proportional to the cube of the total quantum number

${ }^{26}$ Sugiura, J. de Phys., 8, p. 113; 1927.

27 Waible, Zeits. f. Phys., 5s, p. 459; 1929. 
when the number is large. This is not in satisfactory agreement with our value $k=2.3 \times 10^{-19}$ at the limit. Other measurements ${ }^{28}$ on the first four lines of caesium where lines were broadened by high pressures of an inert gas give values about ten times greater than Waible's. From the same source $k$ for other alkalies can be computed with the following results: $\mathrm{Na}$ (Trumpy) $8 \times 10^{-19}, \mathrm{Rb} 5 \times 10^{-19}$.

Various theoretical derivations of the absorption beyond series limits of the hydrogen atom give values which are about a hundred times greater than our value for $1 S$ of caesium. Thus, Oppenheimer ${ }^{29}$ gives for hydrogen in the $2_{2}$ state

$$
k=0.99 \times 10^{-12} \frac{\lambda^{3.4}}{\lambda_{0}^{2.4}} \quad k=3.6 \times 10^{-17} \text { at } \lambda_{0}
$$

This is quite similar to the result computed from recombination data for caesium atoms in the $2 P$ state $\left(k=3 \times 10^{-17}\right.$ at the limit and decreases as $\lambda^{4}$ ).

Sugiura ${ }^{30}$ gives approximate theoretical computations for sodium line absorption which are in satisfactory agreement with experiment. The theory assumes that all one-electron systems have the same total absorption integrated over all lines and the continuous spectrum, and it is shown that the low absorption beyond the limit is associated with an abnormally high absorption for the first line in the principal series as compared with the hydrogen value.

We are greatly indebted to Doctor Coblentz and to Mr. Stair for generously advising and assisting us in making the radiation measurements and for supplying apparatus and calibrated standards for our work.

\section{ADDENDUM}

Since this work was completed, Lawrence and Edleison have reported before the Physical Society ${ }^{31}$ relative measurements of photoionization in caesium, rubidium, and potassium made by the space-charge method. The results for rubidium are very similar to ours; their caesium curve is more nearly like rubidium. For potassium their measurements show a well-defined peak at the principal series limit in addition to the increase further in the ultra-violet with evidence of a maximum near $2300 \mathrm{~A}$, which is roughly three times the maximum at the series limit.

Washington, May 8, 1929.

\footnotetext{
${ }^{28}$ Wolf and Herzfeld, Handbuch der Phys., 20, p. 616.

29 Oppenheimer, Zeits. f. Phys., 41, p. 268; 1927.

${ }^{30}$ Sugiura, Phil. Mag., 4, p. 495; 1927.

31 Proc. Washington Meeting, Apr. 18-20; 1929.
} 